\title{
FINITE ELEMENT METHODS FOR THE SIMULATION OF INCOMPRESSIBLE POWDER FLOW
}

\author{
J. Hron, A. Ouazzi and S. Turek \\ Institute of Applied Mathematics, University of Dortmund, 44227 Dortmund, Germany
}

\begin{abstract}
The flowing of powders brings a new challenging and interesting problem to the CFD community: at very high concentrations and low rate-of-strain, grains are in permanent contact, rolling on each other. Therefore a frictional stress model must be taken into account. This can be done using plasticity and similar theories in which the granular material behaviour is assumed to be independent of the velocity gradient or the rate-of-strain. This is in contrast to viscous Newtonian flow where the stress specifically depends on the rate-of-strain. Furthermore, unlike fluids, flowing powders do not exhibit viscosity and, again, this shows that a Newtonian rheology cannot accurately describe granular flow. It is assumed that the material is incompressible, dry, cohesionless, and perfectly rigid-plastic. Based on continuum theories, generalized Navier Stokes equations have been derived by replacing the velocity gradient by the shear rate, and the viscosity depends on pressure and shear rate. Thus the resulting equation is mathematically more complex than the Navier Stokes equations and is valid only when the material is deforming. In this note we present numerical algorithms to approximate these highly nonlinear equations. A Newton linearization technique is applied directly to the corresponding continuous variational formulations. The approximation of incompressible velocity fields is treated by using stabilized nonconforming Stokes elements and we use a Pressure Schur Complement smoother as defect correction inside of a direct multigrid approaches to solve the linear saddle-point problems with high numerical efficiency. The results of several computational experiments for realistic flow configurations are provided.
\end{abstract}

\section{PHYSICAL BACKGROUD}

\section{Mohr-Coulomb criterion for friction}

The Mohr theory suggests that the shear stress on a failure reaches some unique function of normal stress, $\tau=f(\sigma)$, where $\tau$ is the shear stress and $\sigma$ is the normal stress. This function can be graphically expressed by the Mohr failure envelope: the tangent of Mohr circles is shown in Figure1 for different $\tau$ and $\sigma$ at a failure. The Mohr failure hypothesis states that the point of tangency of the Mohr failure envelope with the circle at a failure determines the inclination of the failure plane. Coulomb found that there was a stress-independent component of shear strength and a stress dependent component. He called the latter the internal angle of friction, $\phi$, and the former seems to be related to the intrinsic cohesion and is denoted by the symbol $c$. Then, the Coulomb equation reads

$$
\tau=\sigma \tan \phi+c
$$


where $\tau$ is the shear strength of the soil, $\sigma$ is the applied normal stress, and $\phi$ and $c$ are the material constants defined as the cohesive strength and the angel of internal friction, respectively; a material is called non-cohesive if $c=0$. Eq. (1) represents the simple law of friction of two solids sliding on each other with the shear force proportional to the normal force, $\eta=\tan \phi$ being the friction coefficient.

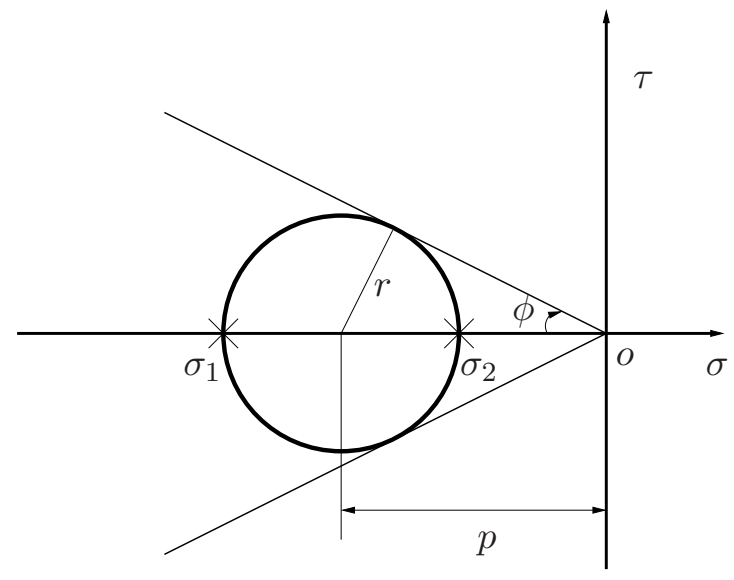

Figure 1: Mohr's circle of stress in $(\sigma, \tau)$-space and yield for a cohesionless granular material with an internal friction angle $\phi$

A similar condition also exists at the interface between the granular material and the walls of the container: only the angle of internal friction is replaced by the angle of wall friction, $\phi_{W}$. The angle satisfies $\phi_{W}<\phi$ since the wall is usually less rough than a powder layer; this is mainly due to the void fraction near the wall.

\section{Regimes of powder flow}

Analogous to the fluid flow, the powder regimes can be represented as a function of a dimensionless shear rate $\gamma^{o *}=\gamma^{o}\left[d_{p} / g\right]^{1 / 2}$ which contains a gravitational term $g$ and the particle size $d_{p}$, and which plays the similar role as the Reynolds number $R e$ for fluids (Tardos et al. [9]). See Fig. 2 for the different regimes.

\subsection{Quasi-static regime}

This regime is valid when the flow is slow enough that any movement between two static states can be neglected; then the static equilibrium equation can be applied. With this approach only stress and condition of the onset of flow can be computed, while no flow field can be predicted which circumscribes the range of applications of this approach. There are a large number of analytical and numerical solutions to this case and an important number of literature devoted to this regime, see for instance [18],[19],[20]. 


\subsection{Slow and frictional regime}

In this regime the frictional forces between particles are predominant, so the inertial effect is added to the static equations as well as the consideration of the continuity beside a yield condition. The first model invoking a flow rule was introduced by Schaeffer (1987) [17]. This regime is very important since it can be used for modeling a wide range of practical phenomenon and industrial applications. However, for the serious challenges which arise in this regime, for instance ill-posed partial differential equations and the prediction of stress fluctuations, there is still a lack of fundamental research so that dealing with these problems requires a multidisciplinary treatment. Our contribution has the goal of supporting this part and will be described in the forthcoming sections.

\subsection{Intermediate and rapid granular regimes}

For the intermediate regime, additional to inter-particle friction energy, collisional energy is important, too. For the rapid regime, the short particle-particle contacts are important while frictional forces are neglected. This regime is often described via kinetic models and will not be treated in this paper. It was reported here just to have a complete view on the different regimes of powder flow (see Figure 2).

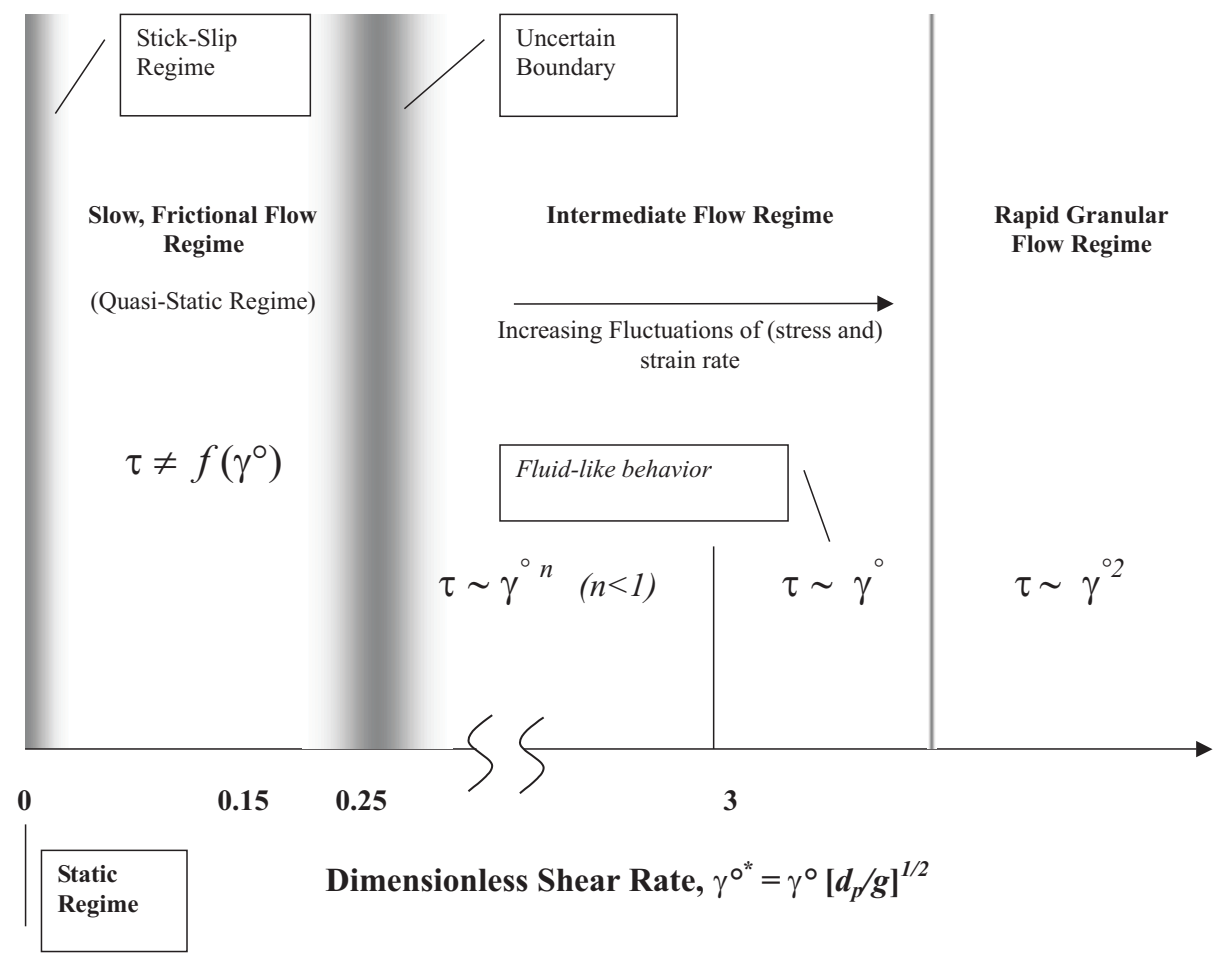

Figure 2: Regimes of powder flow with dimensionless rate $\gamma^{o *}$ (from Tardos [9]) 


\section{Flow rule: Saint Venant principle}

The Saint Venant principle of solid mechanics says that stresses cause deformations preferentially in the same direction. This leads to the co-axiality flow rule condition which states that the principal directions of the stress and rate deformation are parallel and neglect the rotation of a material element during deformation. In two-dimensional Cartesian coordinates, this condition takes the form, for example:

$$
\frac{\mathbf{T}_{x x}-\mathbf{T}_{y y}}{\mathbf{T}_{x y}}=\frac{2(\partial u / \partial x-\partial v / \partial y)}{\partial u / \partial x+\partial v / \partial y}
$$

This was postulated by Schaeffer [17] for the deformation of granular material. However, since the deformation of the granular material requires that the stresses in different directions must be different, Schaeffer claimed that "the response of the material to such unequal stresses should be to contract in the directions of greater stress and to expand in the directions of smaller stress". This reflects the requirement that the eigenvectors of stress tensor and strain rate are aligned and it quantitatively links the deviatoric stress and the strain rate tensor by the following formula:

$$
\mathbf{S}=\lambda \mathbf{D}
$$

\section{Rigid perfect plastic}

\subsection{Plastic deformation}

The deformation of a granular material is considered to be plastic in the sense that, if after deformation the shearing stress is reduced, the material would not show any tendency to return to its original state. Plastic deformation was already proposed by E. C. Bingham, in 1922, in the context of non-Newtonian fluids, and in which the rheological behavior is governed by the following equation introduced by Oldroyd in modified state:

$$
\mathbf{T}=-p I+\left(\frac{\mu_{0}}{\|\mathbf{D}\|}+\mu\right) \mathbf{D}
$$

\subsection{Dilatancy}

A simple manifestation of this phenomenon occurs when one leaves dry footprints while walking along a wet beach: the deformed sand dilates, therefore space between grains increases, allowing for upper water to invade the sand. As a consequence, footsteps get dry and water goes down. This is the phenomenon of dilatancy which was explained by Reynolds in 1885, and demonstrated experimentally: a glass tube attached to a balloon showed that the amount of excess water decreased when the sand was deformed, thus showing that deformation increases the space between grains. Dilatancy is important in the dynamics of granular material, introducing a stick-slip instability at low velocity (see [21]), and it occurs because each grain needs more space in the flowing state than at rest. Then, the flow theory of plasticity must be applied to the constitutive modelling for describing the deformation process of a granular material. 


\section{CONSTITUTIVE EQUATIONS FOR POWDER FLOW}

\section{Equation of motion}

The powder is assumed to be an incompressible continuum that obeys conservation of mass and momentum (density $\rho$, gravity g, velocity $\boldsymbol{u}$ ):

Conservation of mass: With the material derivative $\frac{D *}{D t}$, there holds:

$$
\frac{D \rho}{D t}=\frac{\partial \rho}{\partial t}+\nabla \cdot(\rho \boldsymbol{u})=0
$$

Incompressible material: The bulk density, $\rho$, is a constant, so that

$$
\nabla \cdot \boldsymbol{u}=0 .
$$

Equation of motion: With $\mathbf{T}=\mathbf{S}-p \mathbf{l}$, there holds:

$$
\rho \frac{D \boldsymbol{u}}{D t}=\nabla \cdot \mathbf{T}+\rho \mathbf{g}
$$

\section{Constitutive equations}

The constitutive equation is devoted to correlate between the deviatoric tensor $\mathbf{S}$ and the velocity, through the second invariant of the rate deformation $D_{\mathbb{I}}=\frac{1}{2} \mathbf{D}: \mathbf{D}$, where the rate of deformation is given by $\mathbf{D}=\frac{1}{2}\left(\nabla \boldsymbol{u}+\nabla^{T} \boldsymbol{u}\right)$. There are several examples:

Newtonian law: $\mathbf{S}=2 \nu_{0} \mathbf{D}$

Power law: $\mathbf{S}=2 \nu\left(D_{\mathbb{I}}\right) \mathbf{D}, \quad \nu(z)=z^{\frac{r}{2}-1}, \quad r \geq 1 \quad(r=1$ : Bingham law $)$

Schaeffer's law: For a powder, a constitutive equation was first introduced by Schaeffer [17] which has to obey a

- yield condition: $\|\mathbf{S}\|=\sqrt{2} p \sin \phi$,

- flow rule: $\mathbf{S}=\lambda \mathbf{D}$ with $\lambda \geq 0$.

In fact, the flow rule is based on a yield criterion for granular materials of von Mises type, which is basically derived from a law of sliding friction applied to the individual particles. Specifically in terms of the principal stresses $\sigma_{i}$, this condition is written as

$$
\sum_{i=1}^{3}\left(\sigma_{i}-p\right)^{2} \leq k^{2} p^{2} \quad \text { with } \quad p=\frac{1}{3} \operatorname{tr} \mathbf{T}
$$

where $k=\sqrt{2} \sin \phi$ is a characteristic constant of the material, and $\sigma_{i}$ are the eigenvectors of $\mathbf{T}_{i j}$. For a material that deforms plastically, equality must hold in Eq. (8):

$$
\sum_{i=1}^{3}\left(\sigma_{i}-p\right)^{2}=k^{2} p^{2}
$$


Under plane strain $p=\frac{1}{2}\left(\sigma_{1}+\sigma_{2}\right)$, we may consider a strictly 2D-yield condition:

$$
\left(\sigma_{1}-p\right)^{2}+\left(\sigma_{2}-p\right)^{2}=2 p^{2} \sin ^{2} \phi
$$

A constitutive equation related between stress and strain rate was proposed for slow powder flow by David Schaeffer [17]. This equation obeys the von Mises yield condition and the flow rule (3)

$$
\mathbf{T}=-p I+\sqrt{2} p \sin \phi \frac{\mathbf{D}}{\|\mathbf{D}\|} \quad \text { if } D \neq 0
$$

In fact, the flow rule is assumed to have the form

$$
\mathbf{T}=-p I+\lambda \mathbf{D}
$$

where $\lambda$ is a coefficient. To satisfy the yield condition of flow rule (3) in terms of von Mises, i.e. $\|\mathbf{S}\|=\sqrt{2} p \sin \phi$, then there must hold:

$$
\lambda=\frac{\sqrt{2} p \sin \phi}{\|\mathbf{D}\|}
$$

We use this correlation to obtain finally the constitutive equation $\mathbf{T}=-p I+\sqrt{2} p \sin \phi \frac{\mathbf{D}}{\epsilon+\|\mathbf{D}\|}$, where $\epsilon$ is a typical (small) regularization parameter.

\section{Generalized Navier-Stokes equations}

The derived problem formulation can be stated in the framework of the generalized incompressible Navier-Stokes equations (we set $\rho=1$ ):

$$
\frac{D \boldsymbol{u}}{D t}=-\nabla p+\nabla \cdot\left(\nu\left(p, D_{\mathbb{I}}\right) \mathbf{D}\right)+\mathbf{g}, \quad \nabla \cdot \boldsymbol{u}=0
$$

If we define the nonlinear pseudo viscosity $\nu(\cdot, \cdot)$ as a function of the second invariant of the rate deformation $D_{\mathbb{I I}}$ and the 'pressure' $p$, we can show that various materials can be ranged within different viscosity laws including powder:

- Power law defined for $\nu(z, p)=\nu_{\mathrm{o}} z^{\frac{r}{2}-1}$

- Bingham law defined for $\nu(z, p)=\nu_{\mathrm{o}} z^{-\frac{1}{2}}$

- Schaeffer's law (including the 'pressure') defined for $\nu(z, p)=\sqrt{2} \sin \phi p z^{-\frac{1}{2}}$

A comparison of this equation with the Navier-Stokes equations reveals that the ordinary viscous terms, proportional to the viscosity and the velocity gradient, have been replaced by shearrate independent terms which is quite remarkable, since it implies that an overall increase in the velocity leaves the stress unchanged. This also means that these equations are mathematically more complex than the Navier-Stokes equations and apply only when the material is deforming, for instance for granular flows in hoppers; these equations are only valid for the mass flow where the material is flowing throughout the hopper. 


\subsection{Mathematical challenges}

The main mathematical problems of the incompressible powder model (see Eq. (14)) can be summarized in the following aspects:

- Mathematical analysis: There is a lack of research concerning the existence and regularity of solutions for the flow of such fluids except for special cases (see for instance $[5,8]$ ); furthermore the dynamic equations (14) show some instabilities (see Schaeffer in $[16,17])$.

- Singular viscosity: The extra part, $\sqrt{2} p \sin \phi \frac{\mathbf{D}}{\|\mathbf{D}\|}$, of the stress tensor $\mathbf{T}$ is well defined only for non zero values of the rate of strain tensor and for positive pressure. Therefore, some stabilization techniques of singular phenomena due to nonlinear viscosity are required. For the pressure, one may think about using a positive viscosity function in the pressure variable, described by $\exp (\beta p)$, and a small regularisation parameter $\epsilon$, that means $\frac{p}{\epsilon+\|\mathbf{D}\|}$ or $\frac{\exp (\beta p)}{\epsilon+\|\mathbf{D}\|}$.

- Discretization method: It is well known that the computation of solutions to such incompressible systems requires that special care is taken in the choice of the approximating spaces in order to make the discrete problem well posed (LBB-condition, for instance). Moreover, since a large number of FEM spaces satisfying the above condition are nonconforming, which however present a locking phenomenon for problems involving the rate of deformation tensor ('Korn's inequality'), some consistent stabilization term is required.

- Nonlinear solver: For this highly nonlinear problem coupling the pressure and velocity even in the viscous term, there is almost no alternative to Newton techniques for treating such nonlinear viscosities.

- Linear multigrid solver: Efficient multigrid methods for saddle-point problems are required which however are nonsymmetric due to the Newton linearization and which contain a variable viscosity function.

Some of the above points will be discussed in the next sections; for more details see [13].

\section{ILL POSEDNESS OF THE INCOMPRESSIBLE GRANULAR FLOW EQUATIONS BASED ON THE SCHAEFFER MODEL}

The material in this section is originally given by Schaeffer [17]. We report here in a compact form the analysis of instabilities concerning general properties of the partial equations analogous to the classification of equations into elliptic, hyperbolic and parabolic. The test for linear instability was performed using the mode analysis and looking for an eigenvalue with a positive real part for a solution with exponential dependence:

$$
e^{i(\xi, x)+\lambda(\xi) t}
$$


Stokes equation: For a guidance of analysis, let us consider the linearized equations of Stokes equations:

$$
\left(\begin{array}{cc}
-\mu|\xi|^{2} I & \xi \\
\xi^{T} & 0
\end{array}\right)\left(\begin{array}{l}
\boldsymbol{u} \\
p
\end{array}\right)=\lambda\left(\begin{array}{l}
\boldsymbol{u} \\
0
\end{array}\right)
$$

The eigenvalue $\lambda$ is then obtained as

$$
\lambda=-\mu|\xi|^{2}
$$

which means that the Stokes equations are linearly well-posed.

Granular case: In contrast the linearized equations of motion of the granular case lead to the generalized eigenvalue problem [17]

$$
\left(\begin{array}{cc}
-\frac{k}{\|\mathbf{D}\|}\left[\frac{|\xi|^{2}}{2} I+(A \xi)(A \xi)^{T}\right] & (I+k A) \xi \\
\xi^{T} & 0
\end{array}\right)\left(\begin{array}{c}
\boldsymbol{u} \\
p
\end{array}\right)=\lambda\left(\begin{array}{c}
\boldsymbol{u} \\
0
\end{array}\right)
$$

where $A=\mathbf{D} /\|\mathbf{D}\|$ and $k=\sqrt{2} \sin \phi$. Similarly computing $\lambda$ as before, one obtains:

$$
\lambda=-\frac{\sqrt{2} \sin \phi}{\|\mathbf{D}\|}\left[\frac{(A \xi, \xi)^{2}-\frac{1}{2}|\xi|^{2}(A \xi, \xi)}{|\xi|^{2}+\sqrt{2} \sin \phi(A \xi, \xi)}\right]
$$

The presence of the factor $(A \xi, \xi)$ leads to an indefinite quadratic form in the numerator since $\operatorname{tr} A=0$ and $\operatorname{tr} A^{2}=1$, so that one can conclude that the two-dimensional granular flow equations are linearly ill posed (see [17] for more details).

\section{FEM TECHNIQUES FOR THE NUMERICAL SIMULATIONS}

The range of practical real world problems which involve granular materials is growing, and since the considered problems become more complex and experimentally more expensive, one is particularly interested in the development of new and more powerful computational methods for solving these problems numerically.

\section{Problem formulation}

Let us consider the flow of the stationary generalized Navier-Stokes problem in (14) in a bounded domain $\Omega \subset \mathbb{R}^{2}$. If we restrict the set $V$ of test functions to be divergence-free and if we take the constitutive laws into account, the above equations from (14) lead to:

$$
\int_{\Omega} 2 \nu\left(D_{\mathbb{I}}(\boldsymbol{u}), p\right) \mathbf{D}(\boldsymbol{u}): \mathbf{D}(\boldsymbol{v}) d x+\int_{\Omega}(\boldsymbol{u} \cdot \nabla \boldsymbol{u}) \boldsymbol{v} d x=\int_{\Omega} \boldsymbol{f} \boldsymbol{v} d x, \quad \forall \boldsymbol{v} \in V
$$

It is straightforward to penalize the constraint $\operatorname{div} \boldsymbol{v}=0$ to derive the equivalent mixed formulation of (19): 
Find $(\boldsymbol{u}, p) \in X \times M$ (with spaces $X=H_{0}^{1}(\Omega)$ and $M=L^{2}(\Omega)$ ) so that:

$$
\begin{array}{rlrl}
\int_{\Omega} 2 \nu\left(D_{\mathbb{I}}(\boldsymbol{u}), p\right) \mathbf{D}(\boldsymbol{u}): \mathbf{D}(\boldsymbol{v}) d x & +\int_{\Omega}(\boldsymbol{u} \cdot \nabla \boldsymbol{u}) v d x & +\int_{\Omega} p \operatorname{div} \boldsymbol{v} d x \\
& =\int_{\Omega} \boldsymbol{f} \boldsymbol{v} d x, & & \forall \boldsymbol{v} \in X \\
\int_{\Omega} q \operatorname{div} \boldsymbol{u} d x & =0, & & \forall q \in M
\end{array}
$$

\section{Finite element method discretization}

We consider a subdivision $T \in \mathcal{T}_{h}$ consisting of quadrilaterals in the domain $\Omega_{h} \subset \mathbb{R}^{2}$, and we employ the nonconforming rotated bilinear Rannacher-Turek element [7]. For any quadrilateral $T$, let $(\xi, \eta)$ denote a local coordinate system obtained by joining the midpoints of the opposing faces of $T$. Then, in the nonparametric case, we set on each element $T$

$$
\tilde{Q}_{1}(T):=\operatorname{span}\left\{1, \xi, \eta, \xi^{2}-\eta^{2}\right\} .
$$

The degrees of freedom are determined by the nodal functionals $\left\{F_{\Gamma}^{(a, b)}(\cdot), \Gamma \subset \partial \mathcal{T}_{h}\right\}$,

$$
F_{\Gamma}^{a}:=|\Gamma|^{-1} \int_{\Gamma} v d \gamma \quad \text { or } \quad F_{\Gamma}^{b}:=v\left(m_{\Gamma}\right) \quad\left(m_{\Gamma} \text { midpoint of edge } \Gamma\right)
$$

such that the finite element space can be written as

$$
\begin{aligned}
& W_{h}^{a, b}:=\left\{v \in L_{2}\left(\Omega_{h}\right), v \in \tilde{Q}_{1}(T), \forall T \in \mathcal{T}_{h}, v\right. \text { continuous w.r.t. all } \\
&\text { nodal functionals } \left.F_{\Gamma_{i, j}}^{a, b}(\cdot), \text { and } F_{\Gamma_{i 0}}^{a, b}(v)=0, \forall \Gamma_{i 0}\right\} .
\end{aligned}
$$

Here, $\Gamma_{i, j}$ denote all inner edges sharing the two elements $i$ and $j$, while $\Gamma_{i 0}$ denote the boundary edges of $\partial \Omega_{h}$. In this paper, we always employ version 'a' with the integral mean values as degrees of freedom. Then, the corresponding discrete functions will be approximated in the spaces

$$
V_{h}:=W_{h}^{a} \times W_{h}^{a}, L_{h}:=\left\{q_{h} \in L^{2}(\Omega), q_{h \mid T}=\text { const. }, \forall \mathcal{T} \in \mathcal{T}_{h}\right\} .
$$

Due to the nonconformity of the discrete velocities, the classical discrete 'Korn's Inequality' is not satisfied which is important for problems involving the symmetric part of the gradient [6]. Therefore, appropriate edge-oriented stabilization techniques (see $[2,3,12,15]$ ) have to be included which directly treat the jump across the inter-elementary boundaries via adding the following bilinear form

$$
\sum_{\text {edges } E} \frac{1}{|E|} \int_{E}\left[\phi_{i}\right]\left[\phi_{j}\right] d \sigma
$$

for all basis functions $\phi_{i}$ and $\phi_{j}$ of $V_{h}$. Taking into account an additional relaxation parameter $s=s(\nu)$, the corresponding stiffness matrix $S$ is defined via:

$$
\langle S \boldsymbol{u}, \boldsymbol{v}\rangle=s \sum_{E \in E_{I} \cup E_{D}} \frac{1}{|E|} \int_{E}[\boldsymbol{u}][\boldsymbol{v}] d \sigma
$$


Here, the jump of a function $\boldsymbol{u}$ on an edge $\mathrm{E}$ is given by

$$
[\boldsymbol{u}]= \begin{cases}\boldsymbol{u}^{+} \cdot \boldsymbol{n}^{+}+\boldsymbol{u}^{-} \cdot \boldsymbol{n}^{-} & \text {on internal edges } E_{I} \\ \boldsymbol{u} \cdot \boldsymbol{n} & \text { on Dirichlet boundary edges } E_{D} \\ 0 & \text { on Neumann boundary edges } E_{N}\end{cases}
$$

where $\boldsymbol{n}$ is the outward normal to the edge and $(\cdot)^{+}$and $(\cdot)^{-}$indicate the value of the generic quantity $(\cdot)$ on the two elements sharing the same edge.

\section{Nonlinear solver: Newton iteration}

In this approach, the nonlinearity is first handled on the continuous level. Let $\boldsymbol{u}^{l}$ be the initial state, then the (continuous) Newton method consists of finding $\boldsymbol{u} \in V$ such that

$$
\begin{aligned}
\int_{\Omega} 2 \nu\left(D_{\mathbb{I}}\left(\boldsymbol{u}^{l}\right), p^{l}\right) \mathbf{D}(\boldsymbol{u}): \mathbf{D}(\boldsymbol{v}) d x & \\
+\int_{\Omega} 2 \partial_{1} \nu\left(D_{\mathbb{I}}\left(\boldsymbol{u}^{l}\right), p^{l}\right)\left[\mathbf{D}\left(\boldsymbol{u}^{l}\right): \mathbf{D}(\boldsymbol{u})\right]\left[\mathbf{D}\left(\boldsymbol{u}^{l}\right): \mathbf{D}(\boldsymbol{v})\right] d x & \\
+\int_{\Omega} 2 \partial_{2} \nu\left(D_{\mathbb{I}}\left(\boldsymbol{u}^{l}\right), p^{l}\right)\left[\mathbf{D}\left(\boldsymbol{u}^{l}\right): \mathbf{D}(\boldsymbol{v})\right] p d x & \\
& =\int_{\Omega} \boldsymbol{f} \boldsymbol{v}-\int_{\Omega} 2 \nu\left(D_{\mathbb{I}}\left(\boldsymbol{u}^{l}\right), p^{l}\right) \mathbf{D}\left(\boldsymbol{u}^{l}\right): \mathbf{D}(\boldsymbol{v}) d x, \quad \forall \boldsymbol{v} \in V
\end{aligned}
$$

where $\partial_{i} \nu(\cdot, \cdot), i=1,2$, is the partial derivative of $\nu$ related to the first and second variables, respectively; for more details see $[13,14]$.

\section{New linear auxiliary problem}

The resulting auxiliary subproblems in each Newton step consist of finding $(\boldsymbol{u}, p) \in X \times M$ as solutions of the linear (discretized) systems

$$
\begin{cases}A\left(\boldsymbol{u}^{l}, p^{l}\right) \boldsymbol{u}+\delta_{d} A^{*}\left(\boldsymbol{u}^{l}, p^{l}\right) \boldsymbol{u}+B p+\delta_{p} B^{*}\left(\boldsymbol{u}^{l}, p^{l}\right) p & =R_{u}\left(\boldsymbol{u}^{l}, p^{l}\right) \\ B^{T} \boldsymbol{u} & =R_{p}\left(\boldsymbol{u}^{l}, p^{l}\right)\end{cases}
$$

where $R_{u}(\cdot, \cdot)$ and $R_{p}(\cdot, \cdot)$ denote the corresponding nonlinear residual terms for the momentum and continuity equations, and the operators $A\left(\boldsymbol{u}^{l}, p^{l}\right), B, A^{*}\left(\boldsymbol{u}^{l}, p^{l}\right)$ and $B^{*}\left(\boldsymbol{u}^{l}, p^{l}\right)$ are defined as follows:

$$
\begin{gathered}
\left\langle A\left(\boldsymbol{u}^{l}, p^{l}\right) \boldsymbol{u}, \boldsymbol{v}\right\rangle=\int_{\Omega} 2 \nu\left(D_{\mathbb{I}}(\boldsymbol{u}), p\right) \mathbf{D}(\boldsymbol{u}): \mathbf{D}(\boldsymbol{v}) d x \\
\langle B p, \boldsymbol{v}\rangle=\int_{\Omega} p \nabla \cdot \boldsymbol{v} d x \\
\left\langle A^{*}\left(\boldsymbol{u}^{l}, p^{l}\right) \boldsymbol{u}, \boldsymbol{v}\right\rangle=\int_{\Omega} 2 \partial_{1} \nu\left(D_{\mathbb{I}}\left(\boldsymbol{u}^{l}\right), p^{l}\right)\left[D\left(\boldsymbol{u}^{l}\right): D(\boldsymbol{u})\right]\left[D\left(\boldsymbol{u}^{l}\right): D(\boldsymbol{v})\right] d x \\
\left\langle B^{*}\left(\boldsymbol{u}^{l}, p^{l}\right) \boldsymbol{v}, p\right\rangle=\int_{\Omega} 2 \partial_{2} \nu\left(D_{\mathbb{I}}\left(\boldsymbol{u}^{l}\right), p^{l}\right)\left[D\left(\boldsymbol{u}^{l}\right): D(\boldsymbol{v})\right] p d x
\end{gathered}
$$


Some remarks: The full Newton method is performed for $\delta_{d}=1$ and $\delta_{p}=1$, while the fixed point method corresponds to $\delta_{d}=0$ and $\delta_{p}=0$. For $\delta_{d} \neq 0$, the linear problem behaves similar as the classical saddle point problem for power law models, that means with variable viscosity, while for $\delta_{p} \neq 0$ we obtain a new type of problem, involving the nonsymmetric pressure matrix $B^{*}$; for more details, see $[14,13,4]$.

\section{Linear solver}

This section is devoted to give a brief description of the involved solution techniques for the resulting linear systems. For the nonconforming Stokes element $\tilde{Q}_{1} / Q_{0}$, a 'local pressure Schur complement' preconditioner (see [11]) as generalization of so-called 'Vanka smoothers' is constructed on patches $\Omega_{i}$ which are ensembles of one or several mesh cells, and this local preconditioner is embedded as global smoother into an outer block Jacobi/Gauss-Seidel iteration which acts directly on the coupled systems of generalized Stokes, resp., Oseen type as described in [12]. If we denote by $\tilde{R}_{u}$ and $\tilde{R}_{p}$ the discrete residuals for the momentum and continuity equation which include the complete stabilisation term due to the modified bilinear form $S$ in (26), one smoothing step in defect-correction notation can be described as

$$
\left[\begin{array}{c}
\boldsymbol{u}^{l+1} \\
p^{l+1}
\end{array}\right]=\left[\begin{array}{c}
\boldsymbol{u}^{l} \\
p^{l}
\end{array}\right]+\omega^{l} \sum_{i}\left(\begin{array}{cc}
F+S_{\mid \Omega_{i}}^{*} & \tilde{B}+\delta_{p} \tilde{B}_{\mid \Omega_{i}}^{*} \\
\tilde{B}_{\mid \Omega_{i}}^{T} & 0
\end{array}\right)^{-1}\left[\begin{array}{c}
\tilde{R}_{u}\left(\boldsymbol{u}^{l}, p^{l}\right) \\
\tilde{R}_{p}\left(\boldsymbol{u}^{l}, p^{l}\right)
\end{array}\right]
$$

with matrix $F=\tilde{A}+\delta_{d} \tilde{A}^{*}$ and $\tilde{A}, \tilde{B}, \tilde{A}^{*}$ and $\tilde{B}^{*}$ are the discrete matrices corresponding to the operators in (30), (31), (32) and (33). For the preconditioning step only a part of the matrix, i.e. $F+S^{*}$, is involved such that the original FEM data structure is preserved. All other components in the multigrid approach, that means intergrid transfer, coarse grid correction and coarse grid solver, are the standard ones and are based on the underlying hierarchical mesh hierarchy and the properties of the nonconforming finite elements (see [11] and [12] for the details).

\section{NUMERICAL SIMULATIONS}

The purpose of this section is to show that our numerical approaches based on FEM techniques are well suited to address the illustrated type of nonlinear powder problems and leads to comparative results with related experiments. Therefore, we consider the following two configurations, namely powder flow in a couette device with an obstacle and granular flow in a hopper.

\section{Boundary condition}

The boundary conditions in any incompressible fluid simulation are expressed either in terms of the fluid velocity or the pressure at the boundary, but generaly both of them cannot be used at the same boundary since the velocities are influenced by pressure gradient (see [22]). For the Navier Stokes equations with Dirichlet velocity data, the pressure is unique up to a constant which however can be chosen arbitrarily. In contrast, for the flow of the generalized Navier Stokes equations with pressure dependent viscosity, the choice of fixing the pressure cannot be done by random choice (see Table1). 


\subsection{Wall boundary conditions}

The fundamental assumption in fluid mechanics for flow past solids is a "no-slip" boundary condition, which means that is the tangential component of the fluid velocity equals of the solid at the surface. This well-accepted no-slip boundary condition may not be suitable for highly sheared flow, but the error due to the "no-slip" assumption is relatively small in big systems or if we are more interested in the flow far away from the wall. An alternative and more suitable condition is to apply slip with friction parameter $\beta$ :

$$
\boldsymbol{v} \cdot \tau+\beta^{-1} n \cdot\left(2 \nu\left(D_{\mathbb{I}}, p\right) \mathbf{D}(\boldsymbol{v})-p I\right) \cdot \tau=0 \quad \text { on } \Gamma_{\text {wall }}
$$

Moreover, the closing of the equations is required, because the related Dirichlet problem of Navier Stokes equations is well known to possess no unique pressure solution due to the contraint $\operatorname{div} \boldsymbol{v}=0$. The uniquess is assured by fixing the pressure with the choice of mean pressure to be zero which however cannot be taken for the flow with pressure dependent viscosity, namely the Schaeffer law, since it leads to negative values of the pressure in some parts of the computational domain. The first remedy is to make the choice of mean pressure positive to assure a positive pressure in all regions of the computational domain. However, the question arises of the physical meaning of any choice for the mean pressure to get the closure of the equations with Dirichlet boundary condition since the mean pressure is part of the viscosity and, therefore, influences significantly the global flow behaviour.

\subsection{Inflow and Outflow boundary conditions}

Numerical simulations of flow problems usually require the flow out of one or more boundary parts of the computational domain. At such "outflow" boundaries there arises the question of what constitutes a good boundary condition. The simplest and most commonly used outflow condition is that of a "natural" boundary, see [22] for an overview:

$$
2 \nu\left(D_{\mathbb{I}}, p\right) n \cdot \mathbf{D}(\boldsymbol{v})-p n=0 \quad \text { on } \Gamma_{\text {out }}
$$

This boundary condition represents a smooth continuation of the flow through the boundary and occurs in the variational formulation of problem if one does not prescribe any boundary condition for the velocity at the outlet, known in the literature by the name "natural" or "do nothing" boundary condition. It must be stressed that the "do nothing" outflow boundary condition has no physical basis, rather it is a mathematical statement that may or may not provide the desired flow behavior. Particularly, "do nothing" boundary conditions have proven to lead to very satisfactory results in modeling parallel flows, see e.g., Turek [11, 23], but they must always be viewed with suspicion since they contain the hidden condition that the mean pressure is zero across the outflow boundary. In particular, the condition of mean pressure to be zero across the outflow leads to negative values of the pressure, which causes problems for the numerical simulation of flow with pressure dependent viscosity, namely the Schaeffer model. As a natural remedy for this situation, one may consider a condition in which the mean pressure across the outflow coincides with the atmospheric pressure, that means $p_{a t m}>0$ :

$$
2 \nu\left(D_{\mathbb{I I}}, p\right) n \cdot \mathbf{D}(\boldsymbol{v})-p n=p_{a t m} n \quad \text { on } \Gamma_{\text {out }}
$$

The above examples suggest that the ability to specify a pressure condition at one or more parts of the computational domain is an important aspect. This can be done in terms of prescribed pressure drops with corresponding variational formulations of very general type [22]. 


\section{Drag force in powder flow}

As we described before, granular materials can flow like fluids and resist the motion of objects moving through them. Since this retarding force, known as drag force, is easily measured experimentally for a granular medium in a couette device with an immersed cylinder [1], for this reason we choose this configuration for our computation: Although our simulation is only in $2 \mathrm{D}$, a lot of characteristics of granular flow can be examined numerically.

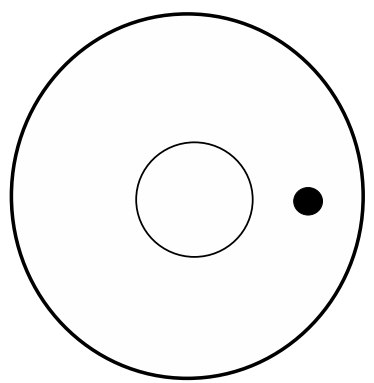

Couette device

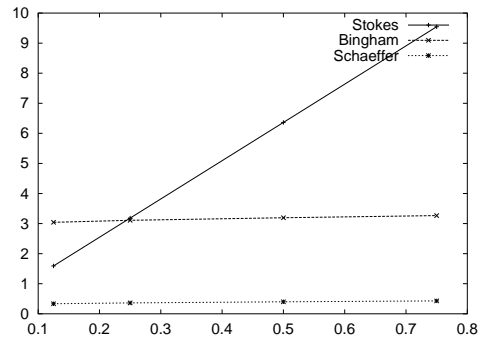

Velocity

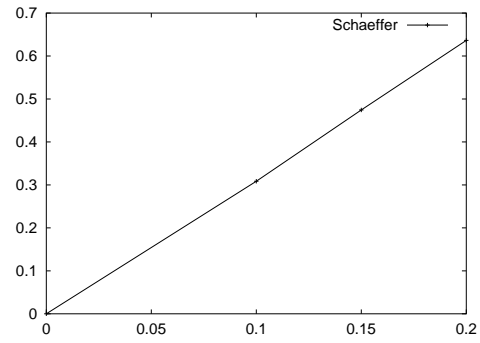

Cylinder diameter

Figure 3: The dependence of drag force with grain velocity in a couette flow around a cylinder for different material laws and for different cylinder diameter

As expected, the drag force for Schaeffer and Bingham flow acting on the cylinder is independent of the grain velocity, contrary to the (newtonian) Stokes flow.

"When mechanical ploughs replaced draught animals, it was observed that ploughing at greater speeds does not require greater forces!”' (Schaeffer 1987)

In Table 1, we show this behaviour more in detail: Here, we perform tests for various prescribed mean pressure values to obtain a unique solution, and for several rotational speeds. As explained before, the Stokes and Bingham model lead to drag forces which are independent of the given mean pressure while the Schaeffer model shows the expected dependence since the pressure is part of the viscous term. However, this observation is only true for low speed. Therefore, we increase continuously the velocity grain and plot the behaviour of the drag force for the Schaeffer model. In Figure 4 we distinguish between three regimes for the Schaeffer model with convection: The first one with slow velocity grain in which the drag force remains constant with increasing speed, the second with intermediate velocity grain, here the drag force shows an exponential increase with the velocity grain and the third one with higher velocity grain in which the drag force shows a linear increase with the velocity grain. In contrast, if we totally neglect the convection term the drag force remains constant with an absolute value which is independent of the velocity grain.

\section{Granular flow in a hopper}

Flow of granular material in hoppers under gravity is quite complex and experimental approaches show limitations in understanding some phenomena for this type of geometry. Our investigation is to understand some typical phenomena related to granular material, namely oscillating phenomena and instabilities, as for instance shear banding instabilities. 


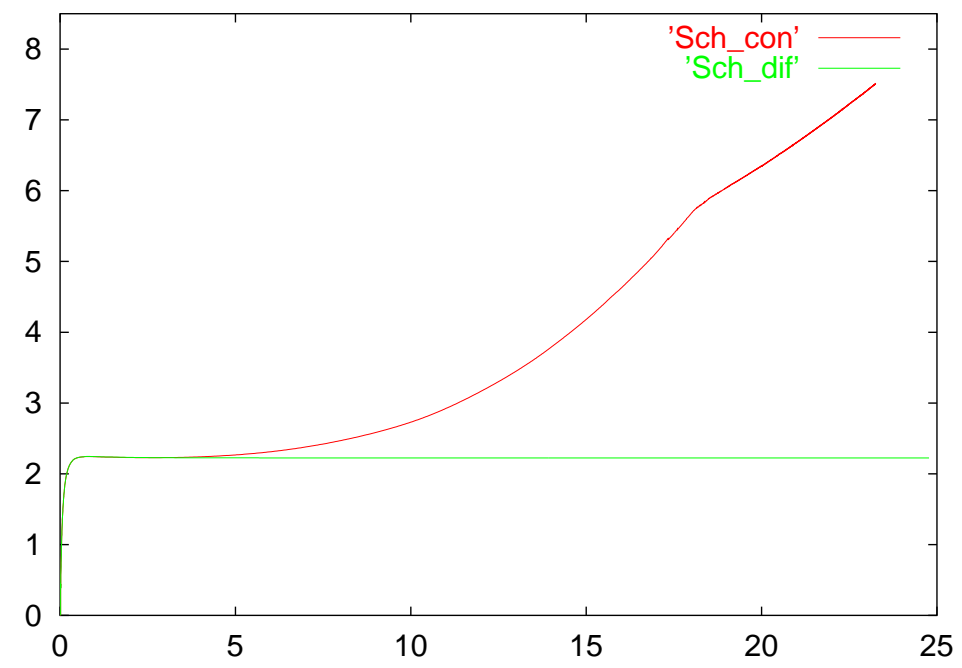

Figure 4: The dependence of the drag force from the velocity grain for the Schaeffer model with ('con') and without ('dif') convection

\begin{tabular}{|c|c|c|c|c|c|}
\hline pressure & 0.5 & 1 & 5 & 10 & 100 \\
\hline \hline Speed & \multicolumn{5}{|c|}{ Stokes law without convection } \\
\hline 0.01 & $0.12575 \mathrm{D}+01$ & $0.12575 \mathrm{D}+01$ & $0.12575 \mathrm{D}+01$ & $0.12575 \mathrm{D}+01$ & $0.12575 \mathrm{D}+01$ \\
\hline 0.05 & $0.62877 \mathrm{D}+01$ & $0.62877 \mathrm{D}+01$ & $0.62877 \mathrm{D}+01$ & $0.62877 \mathrm{D}+01$ & $0.62877 \mathrm{D}+01$ \\
\hline 0.1 & $0.12575 \mathrm{D}+02$ & $0.12575 \mathrm{D}+02$ & $0.12575 \mathrm{D}+02$ & $0.12575 \mathrm{D}+02$ & $0.12575 \mathrm{D}+02$ \\
\hline 0.2 & $0.25151 \mathrm{D}+02$ & $0.25151 \mathrm{D}+02$ & $0.25151 \mathrm{D}+02$ & $0.25151 \mathrm{D}+02$ & $0.25151 \mathrm{D}+02$ \\
\hline 0.4 & $0.50302 \mathrm{D}+02$ & $0.50302 \mathrm{D}+02$ & $0.50302 \mathrm{D}+02$ & $0.50302 \mathrm{D}+02$ & $0.50302 \mathrm{D}+02$ \\
\hline 0.5 & $0.62877 \mathrm{D}+02$ & $0.62877 \mathrm{D}+02$ & $0.62877 \mathrm{D}+02$ & $0.62877 \mathrm{D}+02$ & $0.62877 \mathrm{D}+02$ \\
\hline \hline Speed & \multicolumn{6}{|c|}{ Bingham law without convection } \\
\hline 0.01 & $0.35828 \mathrm{D}+01$ & $0.35828 \mathrm{D}+01$ & $0.35828 \mathrm{D}+01$ & $0.35828 \mathrm{D}+01$ & $0.35828 \mathrm{D}+01$ \\
\hline 0.05 & $0.38137 \mathrm{D}+01$ & $0.38137 \mathrm{D}+01$ & $0.38137 \mathrm{D}+01$ & $0.38137 \mathrm{D}+01$ & $0.38137 \mathrm{D}+01$ \\
\hline 0.1 & $0.38222 \mathrm{D}+01$ & $0.38222 \mathrm{D}+01$ & $0.38222 \mathrm{D}+01$ & $0.38222 \mathrm{D}+01$ & $0.38222 \mathrm{D}+01$ \\
\hline 0.2 & $0.38252 \mathrm{D}+01$ & $0.38252 \mathrm{D}+01$ & $0.38252 \mathrm{D}+01$ & $0.38252 \mathrm{D}+01$ & $0.38252 \mathrm{D}+01$ \\
\hline 0.4 & $0.38264 \mathrm{D}+01$ & $0.38264 \mathrm{D}+01$ & $0.38264 \mathrm{D}+01$ & $0.38264 \mathrm{D}+01$ & $0.38264 \mathrm{D}+01$ \\
\hline 0.5 & $0.38265 \mathrm{D}+01$ & $0.38265 \mathrm{D}+01$ & $0.38265 \mathrm{D}+01$ & $0.38265 \mathrm{D}+01$ & $0.38265 \mathrm{D}+01$ \\
\hline \hline Speed & \multicolumn{7}{|c|}{ Schaeffer law without convection } \\
\hline 0.01 & $0.44745 \mathrm{D}+00$ & $0.89491 \mathrm{D}+00$ & $0.44746 \mathrm{D}+01$ & $0.89492 \mathrm{D}+01$ & $0.89492 \mathrm{D}+02$ \\
\hline 0.05 & $0.50419 \mathrm{D}+00$ & $0.10084 \mathrm{D}+01$ & $0.50419 \mathrm{D}+01$ & $0.10084 \mathrm{D}+02$ & $0.10084 \mathrm{D}+03$ \\
\hline 0.1 & $0.50629 \mathrm{D}+00$ & $0.10126 \mathrm{D}+01$ & $0.50628 \mathrm{D}+01$ & $0.10126 \mathrm{D}+02$ & $0.10126 \mathrm{D}+03$ \\
\hline 0.2 & $0.50756 \mathrm{D}+00$ & $0.10151 \mathrm{D}+01$ & $0.50755 \mathrm{D}+01$ & $0.10151 \mathrm{D}+02$ & $0.10151 \mathrm{D}+03$ \\
\hline 0.4 & $0.50836 \mathrm{D}+00$ & $0.10167 \mathrm{D}+01$ & $0.50835 \mathrm{D}+01$ & $0.10167 \mathrm{D}+02$ & $0.10167 \mathrm{D}+03$ \\
\hline 0.5 & $0.50849 \mathrm{D}+00$ & $0.10170 \mathrm{D}+01$ & $0.50849 \mathrm{D}+01$ & $0.10170 \mathrm{D}+02$ & $0.10170 \mathrm{D}+03$ \\
\hline
\end{tabular}

Table 1: The dependence of the drag force from various rotational velocities at different mean pressure values 

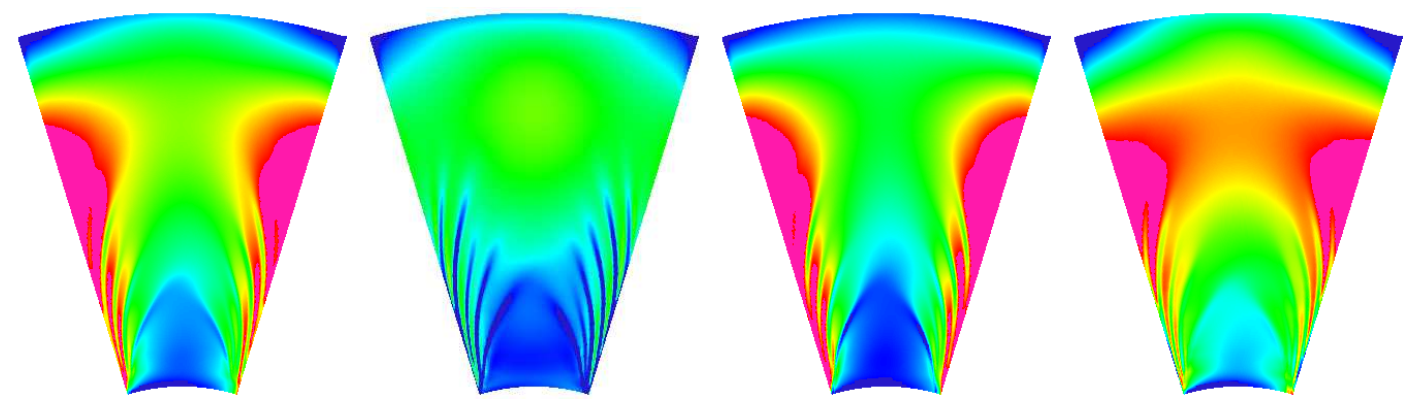

Figure 5: Schaeffer law: The plot of the pressure, the pseudo viscosity, the first and the fourth component of the stress at $\mathrm{t}=0.03 \mathrm{~s}$
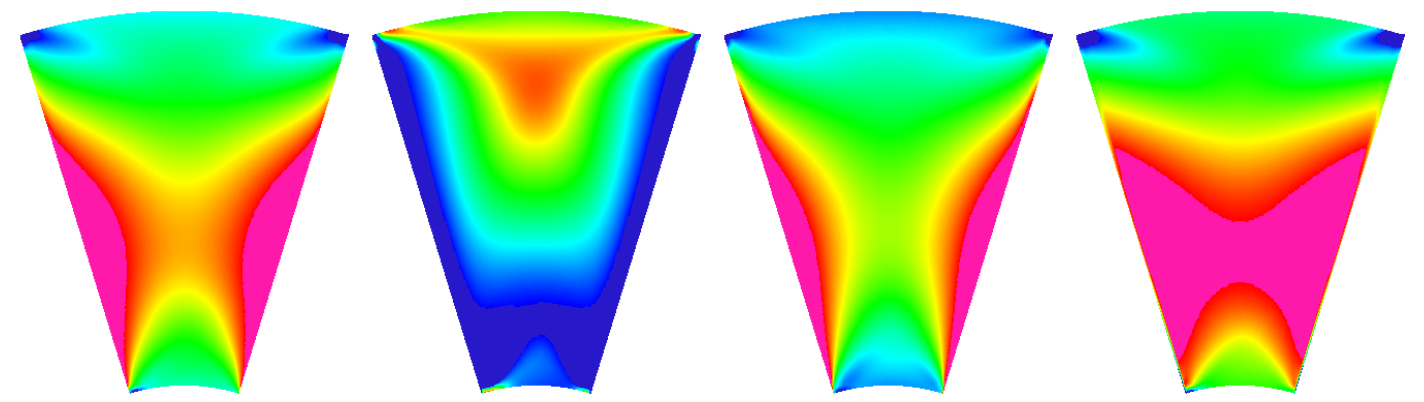

Figure 6: Bingham law: The plot of the pressure, the pseudo viscosity, the first and the fourth component of the stress at $\mathrm{t}=0.03 \mathrm{~s}$

For the Schaeffer model, Figure 5 shows that the flow is significantly influenced by the pressure in the material law, in contrast to the Bingham model (see Figure 6) which is independent of it. To go deeper in understanding the instability phenomena we plot for different times the average stress for both models in Figure 7 and Figure 8. These instabilities may be explained by the stability analysis of Schaeffer [16] who shows the previously illustrated ill-posedness of the problem. However, since we observe that these instabilities arise from the artificial inflow/outflow regions, the influence of the applied boundary conditions is not clear yet. Since the inflow and outflow boundary condition supplied to the hopper do not have any physical meaning, they could be the source of the appearance of the oscilations, too. So, we recently examine a new silo geometry with a long bin on the bottom and the top of the hopper to diminish the influence of the boundary consitions onto the flow behaviour in the constriction.
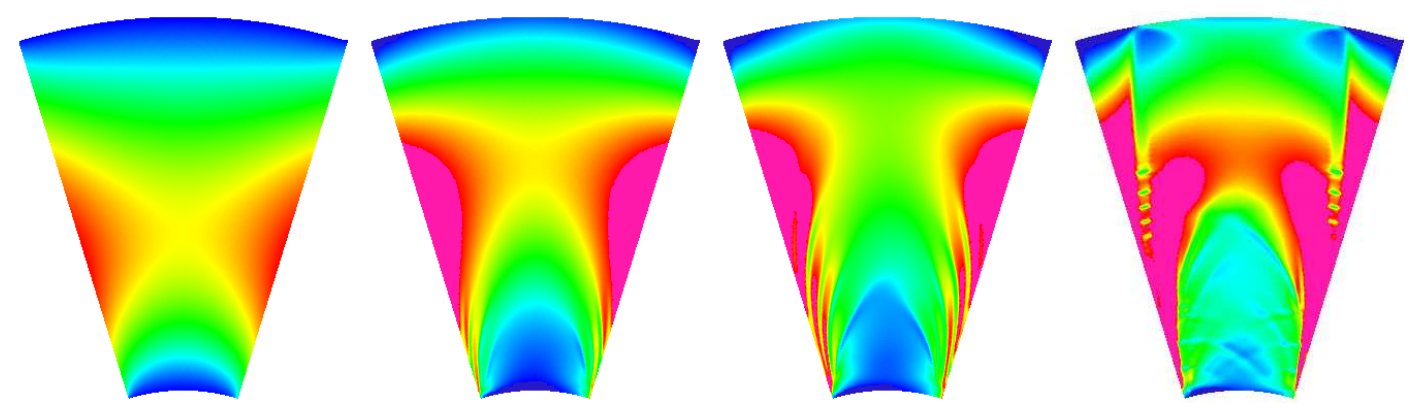

Figure 7: Schaeffer law: Snapshots of the average stress at $t=0.0 \mathrm{~s}, 0.012 \mathrm{~s}, 0.03 \mathrm{~s}$ and $0.06 \mathrm{~s}$ 

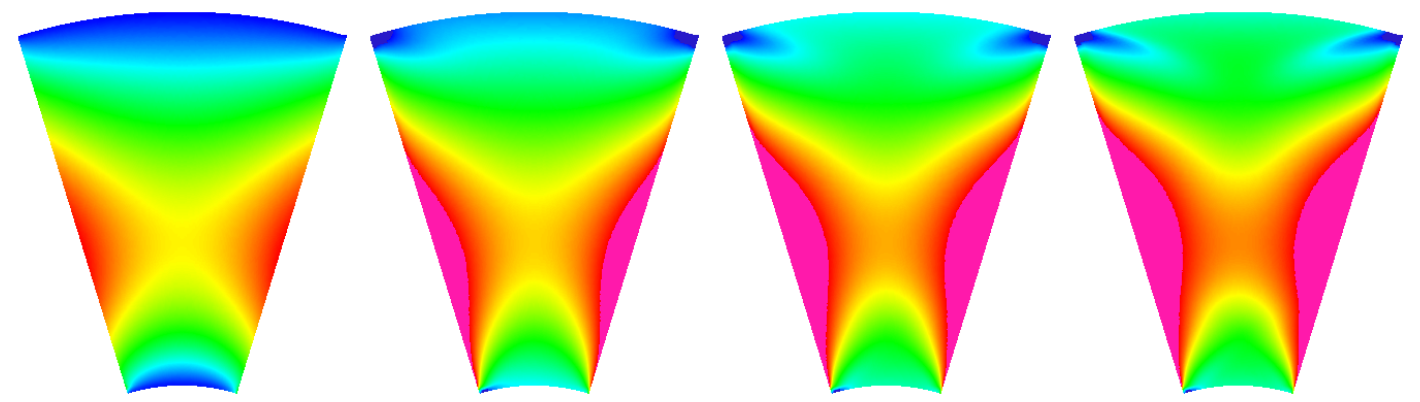

Figure 8: Bingham law: Snapshots of the average stress at $\mathrm{t}=0.0 \mathrm{~s}, 0.012 \mathrm{~s}, 0.03 \mathrm{~s}$ and $0.06 \mathrm{~s}$

\section{SUMMARY}

Our conclusion is that finite element methods together with special material laws are useful tools for the numerical simulation of incompressible granular powder, since the complete structure of the flow is involved, i.e. the velocity, the pressure as well as the stress. Although our computer simulation is only two-dimensional it can confirm well-known physical behavior, namely the independence of the drag force from the velocity grain and the propagation of a pressure wave in the hopper which may lead to a shear banding instability. At first glance, the shear banding phenomenon gives the impression to be treated mathematically as a discontinuity, but this would cause severe problems for numerical algorithms. On the other hand, shear bands might not be a true physical discontinuity, rather than a change in the involved physical system which could be captured with a compressible model.

Furthermore, the proposed incompressible model for granular and powder flow presents some other disadvantages, like for instance the wrong prediction of the flow rate through a symmetric silo by more than a factor of 4 in comparison with experiments. This gives another motivation to proceed toward compressible granular materials which can be specified via the yield condition $q(p, \rho)$ given in Table 2 (see [9] for the details).

\begin{tabular}{|l|l|l|}
\hline Powder properties & Non-cohesive & Cohesive \\
\hline Incompressible & $p \sin \phi$ & $p \sin \phi+c \cos \phi$ \\
\hline Compressible & $p \sin \phi\left[2-\frac{p}{\rho^{\frac{1}{\beta}}}\right]$ & $p \sin \phi \rho^{\frac{1}{\beta}}-C \frac{\left(p-\rho^{\frac{1}{\beta}}\right)^{2}}{\rho^{\frac{1}{\beta}}}$ \\
\hline
\end{tabular}

Table 2: Yield condition for incompressible and compressible powder $(0.001<\beta<0.01)$

Then, the flow can be described by a generalized compressible Navier-Stokes-like equation (38) where a mass conservation equation (39) must hold. However, this is not enough because the density $\rho$ is now a dependent variable, rather than a constant in an incompressible fluid:

$$
\begin{gathered}
\rho \frac{D \boldsymbol{u}}{D t}=-\nabla p+\nabla \cdot\left[\frac{q(p, \rho)}{\left\|\mathbf{D}-\frac{1}{n} \nabla \cdot \boldsymbol{u} I\right\|}\left(\mathbf{D}-\frac{1}{n} \nabla \cdot \boldsymbol{u} I\right)\right]+\rho g \quad n=2,3 \\
\frac{\partial \rho}{\partial t}+\nabla \cdot(\rho \boldsymbol{u})=0
\end{gathered}
$$


In order to complete the system, an additional equation is required in the form of the so-called normality condition:

$$
\nabla \cdot \boldsymbol{u}=\frac{\partial q(p, \rho)}{\partial p}\left\|\mathbf{D}-\frac{1}{n} \nabla \cdot \boldsymbol{u} I\right\|
$$

Since the presented mathematical and computational methodology in this paper can be naturally extended to these compressible granular and powder flow models, our next step is to present the corresponding results and comparisons in forthcoming papers.

\section{References}

[1] Albert, A., Pfeifer, M. A., Brabasi, A. -L., Schiffer, P. (1999), Slow drag in granular medium, Phys. Rev. Lett 82 (1) 205-208

[2] Brenner, C. S. (2004), Korn's inequalities for piecewise $H^{1}$ vector fields, Math. Comp., to appear

[3] Hansbo, P. and Larson, M. G. (2002), Discontinuous Galerkin methods for incompressible and nearly incompressible elasticity by Nitsche's method, Computer Methods in Applied Mechanics an Engineering, 191(17-18), 1895-1908

[4] Hron, J., Ouazzi, A. and Turek, S. (2004) Numerical analysis of FEM techniques and efficient solvers for nonlinear fluids with application to granular and powder flow, in preparation

[5] Hron, J., Malék, J., Necãs, J. and Rajagopal, K. R. (2003), Numerical simulations and global existence of solutions of two-dimensional flows of fluids with pressure- and sheardependent viscosities, Mathematics and Computers in Simulation, 61, Pages 297-315

[6] Knobloch, P. (2000), On Korn's inequality for nonconforming finite elements, Technische Mechanik, 205-214

[7] Rannacher, R. and Turek, S. (1992), A simple nonconforming quadrilateral Stokes element, Numer. Meth. Par. Diff. Eq., 8, 97-111

[8] J. Malék, J. Necãs and K.R. Rajagopal (2002), Global Existence of Solutions for Flows of Fluids with Pressure and Shear Dependent Viscosities Applied Mathematics Letters 15, 961-967

[9] Tardos, G.I., McNamara, S. and Talu, I. (2003), Slow and intermediate flow of a frictional bulk powder in the couette geometry, in press, Powder Technology

[10] Tardos, G.I. (1997), A Fluid Mechanics approach to slow, frictional Powder Flows, Powder Technology, 15, 61-74

[11] Turek, S. (1998), Efficient solvers for incompressible flow problems: An algorithmic and computational approach, Springer, 6, LNCSE

[12] Turek, S., Ouazzi, A. and Schmachtel, R. (2002), Multigrid method for stabilized nonconforming finite elements for incompressible flow involving the deformation tensor formulation, JNM, 10, 235-248 
[13] Ouazzi, A. (2005) Finite element simulation of nonlinear fluids with application to granular material and powder, $\mathrm{PhD}$ thesis, University of Dortmund, to appear

[14] Ouazzi, A. Turek, S. (2003), Numerical Methods and Simulation Techniques for Flow with Shear and Pressure dependent Viscosity, Ergebnisbericht Nr. 241, University of Dortmund

[15] Ouazzi, A. and Turek, S. (2004), Numerical analysis of edge stabilisation for nonconforming finite elements with application to incompressible flow problem, to appear

[16] Schaeffer, D. G. (1997), Mathematical Issues in the continuum formulation of slow granular flow, Springer, New York, 118-129

[17] Schaeffer, D. G. (1987), Instability in the evolution equation describing incompressible granular flow, J. of Differential Equations, 66, 19-50

[18] Skolovskii, V. V. (1956), Static of Granular Material, Oxford, New York

[19] Nedderman, R.M. (1992), Static and Kineamatic of Granular Material,Cambridge Uni. Press, Cambridge, New York

[20] Gremaud, P., Matthews, J.V. (2001), On the Computation of Steady Hopper Flows: I, Stress Determination for Coulomb Materials, with J.V. Matthews, NCSU-CRSC Tech Report CRSC-TR99-35, J. Comput. Phys., 166, p.63-83

[21] Lacombe, F., Zapperi, S., Herrmann, H.J. (1999), Dilatancy and friction in sheared granular media, Eur. Phys. J. E 2, 181-189

[22] Heywood, J. P., Rannacher, R., and Turek, S.(1992), Artificial boundaries and fluxe and pressure conditions for incompressible Navier Stokes equations, Int. J. Numer. Math. Fluids, 22, 325-352

[23] FEATFLOW (1999), Finite element software for the incompressible Navier-Stokes equations: User Manual, Release 1.2 